\title{
Admissible Stress Fields for a Semi-infinite Planar Heap of Granular Medium Possessing Self-weight in Loose Condition*
}

\author{
Sokbil HENG** and Thirapong PIPATPONGSA*** \\ ** Department of International Development Engineering, Tokyo Institute of Technology \\ 2-12-1 O-okayama, Meguro-ku, Tokyo 152-8550, Japan \\ E-mail: hengsokbil@ide.titech.ac.jp \\ ***Global Scientific Information and Computing Center, Tokyo Institute of Technology \\ 2-12-1 O-okayama, Meguro-ku, Tokyo 152-8550, Japan
}

\begin{abstract}
Linear and nonlinear stress propagations in the problem of two-dimensional symmetrical sand pile were investigated. The hyperbolic-type differential equations were formulated under the criterion of self-weight loading. This study shows that the admissible stress solution can be obtained from a wave-like equation by combining the differential equilibrium equations and the local stress conditions with the boundary conditions. Unlike linear stress propagation which appears in straight line, nonlinear stress propagation appears in smooth curves of principal stress directions which are regarded as nests of major and minor arches formed in granular media. The spatial distribution of safety factor against sliding under each closure is also presented and discussed.
\end{abstract}

Key words: Granular Media, Admissible Stress, Stress Propagation, Self-Weight Transfer, Mohr-Coulomb Failure Criteria

\section{Introduction}

Local stress conditions in an ideally granular medium make it possible to obtain the admissible stress fields for many problems. The conditions described in Fig.1 provide the basic assumptions and geometry conditions of semi-infinite planar sand pile. The available choices of local stress conditions in rectangular coordinate system are defined by 4 closures which are constant stress ratio ${ }^{(1),(2),(3)}$, fixed principal axes ${ }^{(4)}$ and oriented stress linearity ${ }^{(5)}$ as well as polarized principal axes ${ }^{(6)}$ which is developed by authors. These solutions give rise to define initial stresses in natural slopes under loose condition. Unlike polarized principal axes, other previous closures are piecewise bi-linear functions; therefore a planar heap is separated into limit state and below-limit state regions with the separated boundary connecting in the stresses of both regions. However, in the closure of polarized principal axes, the limit state regions exist only along the slope surface.

The equilibrium of a granular heap whose sides are free from traction is considered under a planar geometry. Without compaction, granular medium will cascade down once the slope exceeds the angle of repose, thus the heap will grow and reach the unique steady state, with the shape unchanged. Phenomenon of mobilized particles along the slope surfaces was investigated by authors ${ }^{(6)-(8)}$ during establishment of a model heap of loose dry sand on a horizontal rectangular rigid rough base. Model sand heaps were forming in accordance with two distinct methods: sieve source and funnel source ${ }^{(11)}$. According to the appearance

${ }^{*}$ Received 16 Nov., 2009 (No. e68) [DOI: 10.1299/jmmp.4.1261]

Copyright $\odot 2010$ by JSME 
captured during experiments (see Fig.5), the free dropping of particles on the steady slope surfaces inclined with angle of repose creates subsequent sliding surfaces. The failure mechanisms observed on the surface of models sand heap can support the choice of polarized principal axes in analytical model.

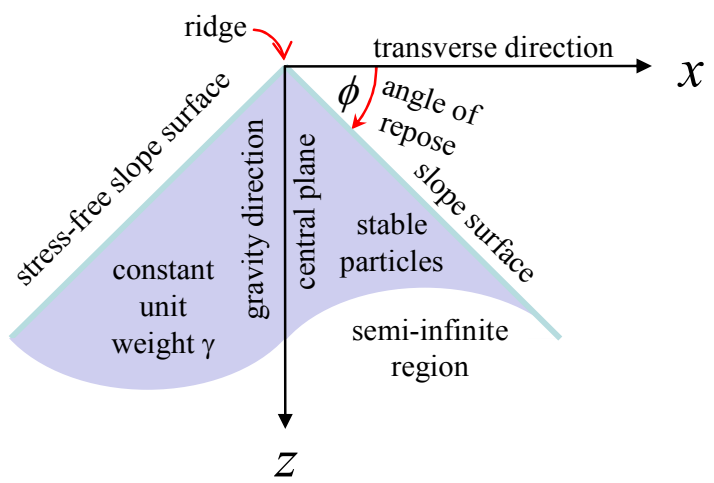

Fig.1 Ideal heap of dry sand in loose condition, forming in a long prismatic shape of semi-infinite planar heap with two symmetrical slopes inclined with angle of repose

\section{Background}

Stress components at a static equilibrium condition of bulk unit weight materials $(\gamma)$, stable under self-weight without surcharge can be represented as follows:

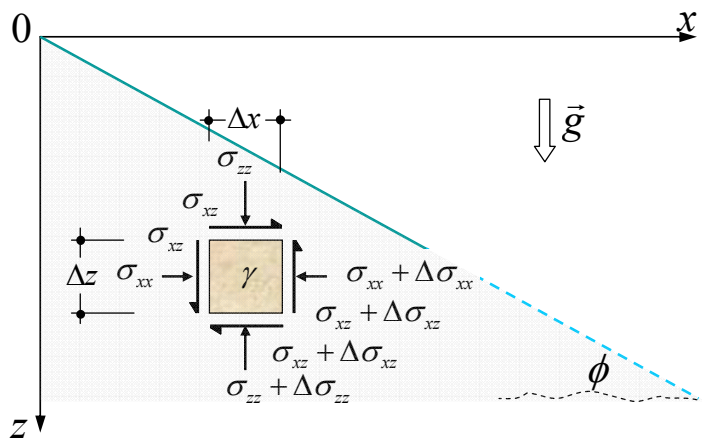

Fig.2 Stress components under static equilibrium condition for a given differential point

From Fig.2, the stress continuity equations in horizontal and vertical directions can be given in the form of implicit functions of first order differential equations $E_{x}$ and $E_{z}$ in which $\sigma_{x x}$, and $\sigma_{z z}$ are normal stresses in horizontal and vertical directions, $\sigma_{x z}=\sigma_{z x}$ is a shear stress, and $\gamma$ is a unit weight.

$$
\begin{aligned}
& E_{x}(x, z)=\partial_{x} \sigma_{x x}+\partial_{z} \sigma_{x z}=0 \\
& E_{z}(x, z)=\partial_{x} \sigma_{x z}+\partial_{z} \sigma_{z z}-\gamma=0
\end{aligned}
$$

The differentiation of implicit Eq.(1) and Eq.(2) with respect to $x$ and $z$ are shown in Eqs.(3) -(4) and Eqs.(5)-(6) respectively.

$$
\begin{aligned}
& \partial_{x} E_{x}(x, z)=\partial_{x x}^{2} \sigma_{x x}+\partial_{z x}^{2} \sigma_{x z}=0 \\
& \partial_{z} E_{x}(x, z)=\partial_{x z}^{2} \sigma_{x x}+\partial_{z z}^{2} \sigma_{x z}=0 \\
& \partial_{x} E_{z}(x, z)=\partial_{x x}^{2} \sigma_{x z}+\partial_{z x}^{2} \sigma_{z z}=0 \\
& \partial_{z} E_{z}(x, z)=\partial_{x z}^{2} \sigma_{x z}+\partial_{z z}^{2} \sigma_{z z}=0
\end{aligned}
$$

Let us formulate a linear combination of differential equations from Eqs.(1)-(6) to the implicit function $\Xi=\Xi(x, z)$, using arbitrary coefficients $a_{1}, a_{2}, a_{3}, a_{4}, a_{7 \mathrm{a}}$ nd $a_{6}$. The value of $\Xi$ 
is zero due to a summation of the differential equations which are regarded as implicit functions.

$$
\Xi(x, z)=a_{1} E_{x}+a_{2} E_{z}+a_{3} \partial_{x} E_{x}+a_{4} \partial_{z} E_{x}+a_{5} \partial_{x} E_{z}+a_{6} \partial_{z} E_{z}=0
$$

Supposing that $\Xi$ is a continuous function, the conditions of $\partial_{k l} \sigma_{i j}=\partial_{l k} \sigma_{i j}=\partial_{k l} \sigma_{j i}$ are satisfied. Consequently, Eq.(7) can be rearranged to Eq.(8), using the following operators.

$$
\begin{aligned}
\Xi(x, z)= & \left(a_{1} \partial_{x}+a_{3} \partial_{x x}^{2}+a_{4} \partial_{x z}^{2}\right) \sigma_{x x}+\left(a_{2} \partial_{x}+a_{1} \partial_{z}+a_{5} \partial_{x x}^{2}+\left(a_{3}+a_{6}\right) \partial_{z x}^{2}+a_{4} \partial_{z z}^{2}\right) \sigma_{x z} \\
& +\left(a_{2} \partial_{z}+a_{5} \partial_{x z}^{2}+a_{6} \partial_{z z}^{2}\right) \sigma_{z z}-a_{2} \gamma=0
\end{aligned}
$$

The above partial differential equation can be solved if a specific stress condition linking all stress components is given.

According to earlier researches listed in Table 1, there are three existing models: $i$ ) BCC, a model assumes horizontal to vertical stress ratio constant throughout the heap body; therefore the horizontal-to-vertical stress ratio $K$ is constant everywhere; ii) FPA, a model assumes principal stress axes fixed at everywhere inside the heap body except the center of heap; therefore, angle of the major and the minor principal stress measured with horizontal axis are fixed from the boundary slope surface; iii) OSL, a model generalizes the two previous models by initiating constant coefficients $\eta$ and $\mu$.

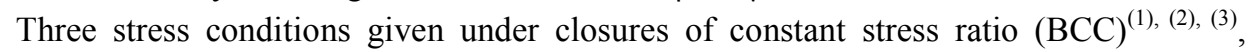
fixed principal axes (FPA) $)^{(4)}$, and oriented stress linearity $(\mathrm{OSL})^{(5)}$ are summarized and listed in Table 1.

Table 1 Various stress conditions in semi-infinite planar heap of granular medium

\begin{tabular}{cc}
\hline Types of closure & Stress conditions \\
\hline Constant stress ratio (BCC) & $\sigma_{x x}=K \sigma_{z z}$ \\
Fixed principal axes (FPA) & $\sigma_{x x}=\sigma_{z z}-2 \sigma_{x z} \tan \phi$ \\
Oriented stress linearity (OSL) & $\sigma_{x x}=\eta \sigma_{z z}+\mu \sigma_{x z}$ \\
\hline
\end{tabular}

By substituting a given relation of stress condition of closure BCC into Eq.(8), for any arbitrary $\sigma_{x z}$, implicit function $\Xi$ can be freed from $\sigma_{x z}$ by selecting $a_{1}=a_{2}=a_{4}=a_{5}=0$ and $a_{3}=-a_{6}$. Providing that $a_{6}$ is an arbitrary coefficient, the following partial differential equation can be obtained.

$$
\begin{aligned}
& \partial_{z z}^{2} \sigma_{z z}-\partial_{x x}^{2}\left(K \sigma_{z z}\right)=\left(\partial_{z z}^{2}-K \partial_{x x}^{2}\right) \sigma_{z z}=0 \\
& \partial_{z z}^{2}\left(\sigma_{x x} / K\right)-\partial_{x x}^{2} \sigma_{x x}=0, \text { or }\left(\partial_{z z}^{2}-K \partial_{x x}^{2}\right) \sigma_{x x}=0
\end{aligned}
$$

For any arbitrary $\sigma_{x x}$ and $\sigma_{z z}$, implicit function $\Xi$ can be freed from $\sigma_{x x}$ and $\sigma_{z z}$ by selecting $a_{1}=a_{2}=a_{3}=a_{6}=0$ and $a_{4}=-(1 / K) a_{5}$. Providing that $a_{5}$ is an arbitrary coefficient, the following partial differential equation can be obtained.

$$
\left(\partial_{x x}^{2}-(1 / K) \partial_{z z}^{2}\right) \sigma_{x z}=0, \text { or }\left(\partial_{z z}^{2}-K \partial_{x x}^{2}\right) \sigma_{x z}=0
$$

Correspondingly, Equations(9), (10), and (11), with manipulation of Eqs.(1)-(2), can formulate a typical form of a second order partial differential equation for a general stress $\sigma_{i j}$, representing any terms of $\sigma_{x x}, \sigma_{x z}$ and $\sigma_{z z}$.

$$
\left(\partial_{z}+c_{1} \partial_{x}\right)\left(\partial_{z}+c_{2} \partial_{x}\right) \sigma_{i j}=\left(\partial_{z z}^{2}+\left(c_{1}+c_{2}\right) \partial_{x z}^{2}+c_{1} c_{2} \partial_{x x}^{2}\right) \sigma_{i j}=0
$$

where $c_{1}$ and $c_{2}$ denote positive and negative coefficients respectively. The values of $c_{1}$ and $c_{2}$ are equivalent to propagation velocities obtained from the boundary conditions. Equation (12) can be classified as a hyperbolic type differential equation because $\left(c_{1}+c_{2}\right)^{2}-4 c_{1} c_{2}>0$. Despite of $c_{1} \neq c_{2}$, Bouchaud et al. ${ }^{(2)}$ observed the expression in Eq.(12) appeared like a wave equation in two dimensions for a physical space $x$ and $z$. For $c=c_{1}=c_{2}$, it becomes an ordinary wave equation. The wave equation is a typical example of a hyperbolic partial differential equation with a parameter $c$ equal to propagation speed of the wave. According 
to Bouchaud et al. ${ }^{(2),(3)}$, the characteristics of the wave-like Eq.(12) can be represented by two straight lines of slope $c_{1}$ and $c_{2}$ which coincide with the principal axes of stresses. Therefore, self-weight transfer is propagated along the major and minor axes of principal stresses which are viewed as load-bearing structures composed of a set of the nested major and minor arches.

The same procedure is applied to the other two closures, FPA and OSL, and the decoupled partial differential equations obtained from the manipulation are summarized in Table 2,

Table 2 Summary of partial differential equations and coefficients of wave-like equation

\begin{tabular}{lll}
\hline \multicolumn{1}{c}{ Stress conditions } & Partial differential equations & Coefficients of wave-like equation \\
\hline Constant stress ratio & $\left(\partial_{z z}^{2}-K \partial_{x x}^{2}\right) \sigma_{i j}=0$ & $c_{1}=\sqrt{K}, c_{2}=-\sqrt{K}$ \\
$\sigma_{x x}=K \sigma_{z z}$ & & $c_{1}=\frac{1-\sin \phi}{\cos \phi}, c_{2}=-\frac{1+\sin \phi}{\cos \phi}$ \\
Fixed principal axes & $\left(\partial_{z z}^{2}-2 \tan \phi \partial_{x z}^{2}-\partial_{x x}^{2}\right) \sigma_{i j}=0$ & \\
$\sigma_{x x}=\sigma_{z z}-2 \sigma_{x z} \tan \phi$ & & $c_{1}=\frac{\mu+\sqrt{\mu^{2}+4 \eta}}{2}, c_{2}=\frac{\mu-\sqrt{\mu^{2}+4 \eta}}{2}$ \\
Oriented stress linearity & $\left(\partial_{z z}^{2}+\mu \partial_{x z}^{2}-\eta \partial_{x x}^{2}\right) \sigma_{i j}=0$ & \\
$\sigma_{x x}=\eta \sigma_{z z}+\mu \sigma_{x z}$ &
\end{tabular}

\section{Model sand heap constructions}

We carried out a series of experiment on sand heap depositing on a rectangular rigid aluminum plate $(70 \mathrm{~cm} \times 30 \mathrm{~cm} \times 1 \mathrm{~cm})$ placing horizontally on a platform. The surface of the plate was stuck with dry sand particles, the same particles sand used for the heap, to form a rough surface. This rectangular rigid aluminum plate with rough surface was used as a vertical support. Two distinct methods of sand heap construction were employed: sieve source (Fig.3a) and funnel source (Fig.3b).

In the first method, dry sand particles dropped by gravity from a sieve and well distributed over the areas of vertical support as a rain dropping. This method is also called sand pluviation method. The deposited sand particles accumulated as a horizontal layer on the vertical support and subsequently buried layer by layer. We elevated the sieve such a way to keep the height of free dropping sand particles constant to each sand layer. As the sand was deposited higher, the perimeter of horizontal burden areas decreases gradually from the surface of vertical support to become almost a straight line at the ridge of the heap, creating 4 side-slope faces of the same angle of repose to the horizontal (Fig.3c).

On the other hand, in the second method, we allowed dry sand particles to drop freely from an opening line of funnel. The deposited sands started to accumulate and form a small shape of heap. We gradually elevated the funnel such a way to keep the dropping height constant from each ridge of sequence wedges of heap. The deposited sand was gradually buried one after another until the heap finally piled up with the base fully occupying the supporting rectangular base, making 4-side-slope faces of the steady heap with the same angle of repose (Fig.3d). We estimate the angle of reposed slope directly by slope measurement tool and verify with undirected measurement using laser pointer projecting shape of slope on to the board (slope height was about $11 \mathrm{~cm}$ ). Both construction methods produced the steady heaps with the same angle of repose at each side and each slope of about 37 degrees. These observation results are consistent with Nadai ${ }^{(10)}$ pointing out that the shape of the heap at a steady state is independent of the method of heap formation. We found out that only the reposed angle $\phi$, which was slightly greater or equal to the internal friction angle of the granular $\left(\phi^{\prime}=36.6^{\circ}\right.$ measured under constant-volume direct box-shear tests, see Fig.4), defines the physical appearance of the heap.

Once the shape of sand heap reaches a steady state, failure surface can occur when the 
dropping of sand particles was continued in the absence of lateral support as shown in Fig.5. We use this failure mechanism to describe the mobilized condition on the slope surface boundary and to assume every particle buried inside the heap body is absolutely under admissible stress condition.

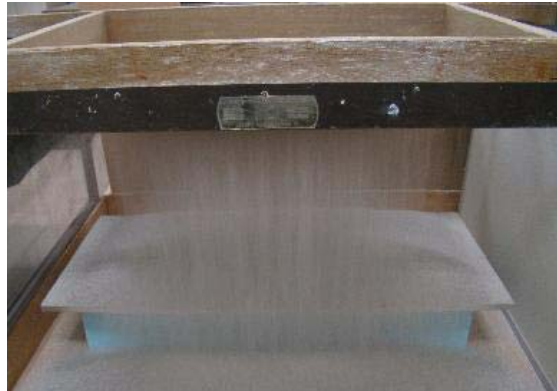

Fig.3a Construction of sand heap by sieve source

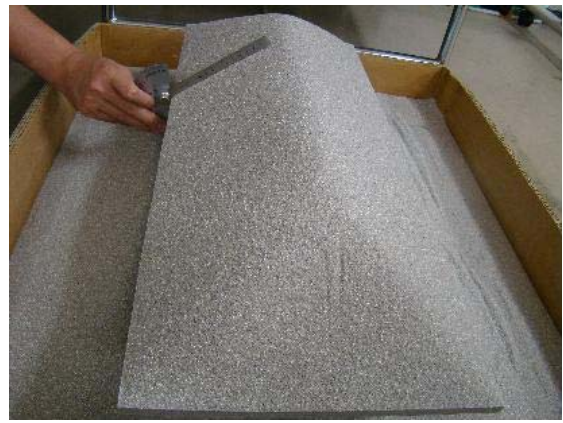

Fig.3c Reposed angle of stable sand heap by sieve source

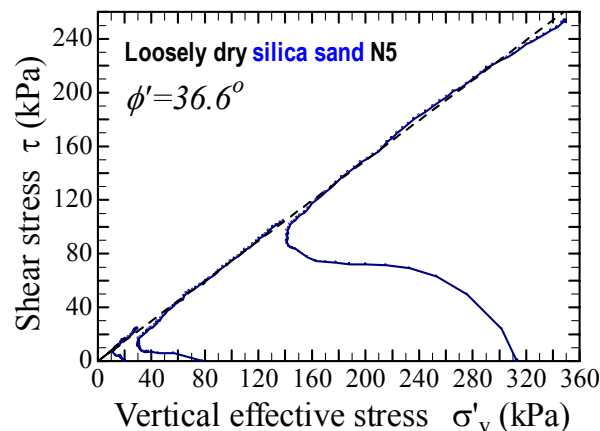

Fig.4 Stress paths during constant volume direct box-shear tests

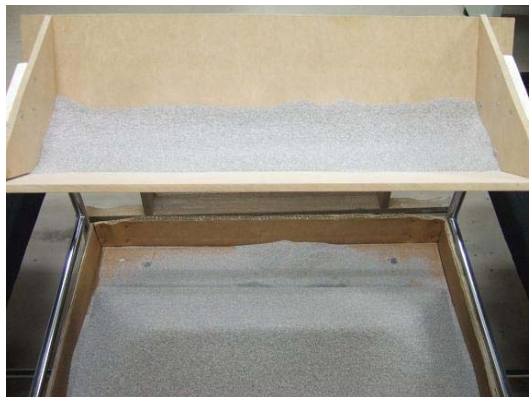

Fig.3b Construction of sand heap by funnel source

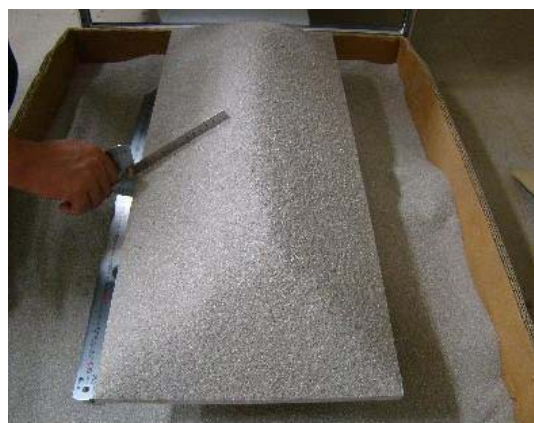

Fig.3d Reposed angle of stable heap by funnel source

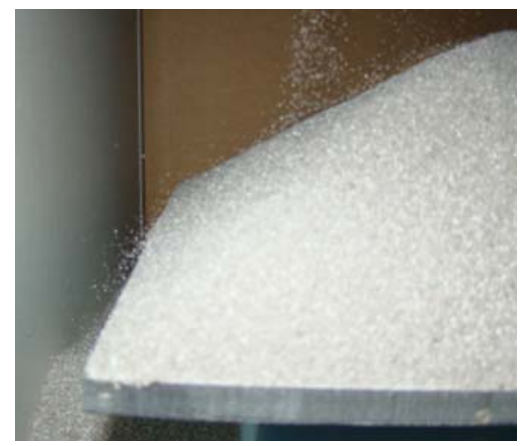

Fig.5 Cascading sand particles at reposed angle slope surfaces

\section{Analytical methods}

Different from previous models, the closure of polarized principal axes (PPA) assumes deeper sand particles lying below the surface of wedge remains stable and immobilized. Only at the slope surface of heap, particles are on the verge of sliding and yielded to ultimate internal friction resistance under the Mohr-Coulomb failure criterion (Fig.6).

Under the assumption of the PPA closure, a principal stress direction measured from transverse direction $\Psi$ is a function of polarized angle $\theta$ (see Fig.6c). The principal stress direction along the failure surfaces $\Psi_{f}$ kept unchanged on both sides of sand heap and found to be $\pi / 4+\phi / 2$ according to Fig. 6 b. The value of $\Psi$ are gradually changed in accordance with its rotation of location described by $\theta$ around the ridge as delineated in Fig.6a. 


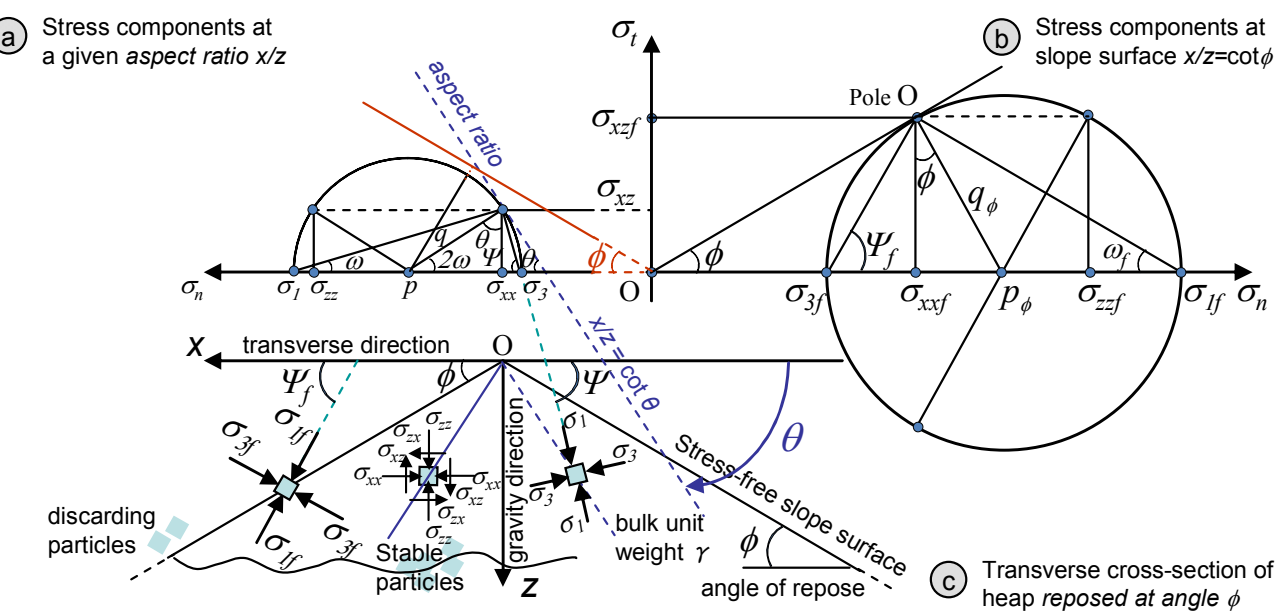

Fig. 6 Mohr's circle representing stresses at a given location in a 2D transverse cross-section of heap

Only at the boundary of traction-free slope surface $\theta=\phi$ whereas inside the wedge body $\theta=\operatorname{acot}(x / z) \geq \phi$; angle of major and minor principal stress measured from horizontal axis are respectively defined from Mohr's circle given in Fig.6a:

$$
\begin{aligned}
& \Psi=\pi / 4+\theta / 2 \\
& \omega=\pi / 4-\theta / 2
\end{aligned}
$$

where $\tan \theta=z / x, \theta \in[\phi, \pi / 2]$

Relation of stress components and the angle of minor principal stress can be given thru Mohr's circle represented in Fig.6a.

$\tan 2 \omega=2 \sigma_{x z} /\left(\sigma_{z z}-\sigma_{x x}\right)$

The major principal stress direction measured horizontal gradually increases whereas minor principal direction decreases from the boundary slope surface. Substitutions of Eqs.(14) and (15) into Eq. (16) can rewrite the relation of local stress condition as:

$$
\sigma_{x x}=\sigma_{z z}-2(z / x) \sigma_{x z}
$$

This local relation of stress condition described by the closure of polarized principal axes $^{(6)}$ can represent the nonlinearity of principal stress orientation.

One might observe that Eq.(17) can be reduced to the closure of fixed principal axes when $z / x=\tan \phi$ is thoroughly kept constant. Eq.(7) substituted by Eq.(17) can remove the components of $\sigma_{x x}$. By enforcing $a_{1}=a_{2}=a_{3}=a_{6}=0$ and $a_{5}=-a_{4}$, further manipulation could be arranged to remove the components of $\sigma_{z z}$, therefore the partial differential for $\sigma_{x z}$ is finally obtained.

$$
-\partial_{x} E_{z}+\partial_{z} E_{x}=\left(\partial_{z z}^{2}-2 \partial_{x z}^{2}(z / x)-\partial_{x x}^{2}\right) \sigma_{x z}=0
$$

Unlike other linear closures, the partial differential equations under the PPA closure can be formulated only for $\sigma_{x z}$. Therefore, once the solution for $\sigma_{x z}$ is found, the solutions for $\sigma_{x x}$, and $\sigma_{z z}$ can be derived from Eqs.(1)-(2) using $\sigma_{x z}$. Further expansion of Eq.(18) can formulate the following equation using arbitrary coefficients $a_{5}=-a_{4}=-\left(x^{2}+z^{2}\right) / x$

$$
\left(\partial_{z z}^{2}-2(z / x) \partial_{x z}^{2}-\partial_{x x}^{2}\right)\left(\left(\left(x^{2}+z^{2}\right) / x\right) \sigma_{x z}\right)=0
$$

which is comparable with a wave-like equation shown previously for a class of linear function. This equation falls into a category of a hyperbolic partial differential equation, which is usually a second-order partial differential equation with discriminant $(z / x)^{2}+1>0$.

\section{Results}

The resulting admissible stress fields can be solved from a wave-like equation with 
stress-free boundary at the slope surface. Detailed solutions could be found in the references (1)-(5). We follow the earlier studies by Sokolovskii ${ }^{(6)}$ by adopting the assumption of similarity solution in which stress distributions have the same shape at all depth. Therefore, stresses can be scaling by unit weight times height of the heap.

$$
\begin{aligned}
& \sigma_{x x}=\gamma z \chi_{x}(x / z) \\
& \sigma_{z z}=\gamma z \chi_{z}(x / z) \\
& \sigma_{x z}=\gamma z \chi_{x z}(x / z)
\end{aligned}
$$

Herein, the implicit functions $\chi_{x}, \chi_{z}, \chi_{x z}$ are solved by the order-reduction technique. Finally with imposed stress conditions at the semi-infinite planar heap boundary (traction free at the slope surface and shear zero at symmetry axis), the particular solutions in term of aspect ratio $x / z$ can be completely derived. Both BCC and FPA closures are piecewise linear functions of aspect ratio $x / z$, having inner elastic core (for $x<\bar{x}$ ) and outer plastic crust (for $x \geq \bar{x}$ ) separated at the relative aspect ratio $\bar{x} / z$ (see Eqs.(27) and (28)). According to Wittmer et al. ${ }^{(4)-(5)}$, the scaling stress functions in the rectangular coordinate system are,

$$
\begin{aligned}
& \chi_{x}(x / z)=\left\{\begin{array}{cc}
(1-(x / z \cot \phi)) \cos ^{2} \phi & \text { if } x / z \geq \bar{x} / z \\
(1-(\bar{x} / z \cot \phi)) \cos ^{2} \phi & \text { otherwise }
\end{array}\right. \\
& \chi_{z}(x / z)=\left\{\begin{array}{cc}
(1-(x / z \cot \phi))\left(1+\sin ^{2} \phi\right) & \text { if } x / z \geq \bar{x} / z \\
1-x / z \cot \phi+(1-1 /(\bar{x} / z \cot \phi))(1-(1+\bar{x} / z \cot \phi)(x / \bar{x})) \sin ^{2} \phi \text { otherwise }
\end{array}\right. \\
& \chi_{x z}(x / z)=\left\{\begin{array}{cc}
(1-(x / z \cot \phi)) \sin \phi \cos \phi & \text { if } x / z \geq \bar{x} / z \\
(x / \bar{x})(1-(\bar{x} / z \cot \phi)) \sin \phi \cos \phi & \text { otherwise }
\end{array}\right.
\end{aligned}
$$

where $\bar{x} / z$ is a function of stress ratio $K=\sigma_{x x} / \sigma_{z z}$ defined at the center of the wedge, expressed by,

$$
\bar{x} / z=\frac{\cot \phi}{2}\left(1-\left(1+2 \tan ^{2} \phi\right) K+\sqrt{(1-K)^{2}+\left(2 K \sin \phi / \cos ^{2} \phi\right)^{2}}\right)
$$

in which the expressions of $\bar{x} / z$ and $K$ for the BCC closure and the FPA closure are presented respectively:

$$
\begin{aligned}
& \bar{x} / z=\cos \phi / \sqrt{1+\sin ^{2} \phi} \text { for } K=\left(1-\sin ^{2} \phi\right) /\left(1+\sin ^{2} \phi\right)=1 /\left(1+2 \tan ^{2} \phi\right) \\
& \bar{x} / z=\cos \phi /(1+\sin \phi) \text { for } K=1
\end{aligned}
$$

According to Pipatpongsa et al. ${ }^{(6)-(8)}$, the particular solution of scale stresses $\chi$ for polarized principal axes closure in term of aspect ratio $x / z$ can be derived:

$$
\begin{aligned}
& \chi_{x}(x / z)=\frac{\tan \phi}{2 \cos ^{2} \phi}\left(\left(3 \phi+\frac{2+\sin ^{2} \phi}{\tan \phi}\right)-\left(3 \cot ^{-1}(x / z)+2 x / z+\frac{x / z}{1+(x / z)^{2}}\right)\right) \\
& \chi_{z}(x / z)=\frac{\tan \phi}{2 \cos ^{2} \phi}\left(\left(\phi+\frac{2-\sin ^{2} \phi}{\tan \phi}\right)-\left(\cot ^{-1}(x / z)+2 x / z-\frac{x / z}{1+(x / z)^{2}}\right)\right) \\
& \chi_{x z}(x / z)=\frac{(x / z \cot \phi)}{2 \cos ^{2} \phi}\left(\left(\cot ^{-1}(x / z)+\frac{x / z}{1+(x / z)^{2}}\right)-\left(\phi+\frac{\sin ^{2} \phi}{\tan \phi}\right)\right)
\end{aligned}
$$

Using the friction angle $\phi=37^{\circ}$ as a material parameter, from Eq.(20)-(22), the general form of stress fields normalized by bulk unit weight $\gamma$ times height of heap $H$ can be simply calculated from the scaling stresses $\chi$ :

$$
\frac{\sigma}{\gamma \mathrm{H}}=\frac{z}{\mathrm{H}} \chi(x / z), \text { where } 0 \leq z / H \leq 1
$$


According to Eq.(32), the color contour of normalized horizontal stresses distribution for $\phi=37^{\circ}$ in a half body and its mirror to the other half along the central line can be illustrated in following figures for the closures of BCC, FPA and PPA respectively.

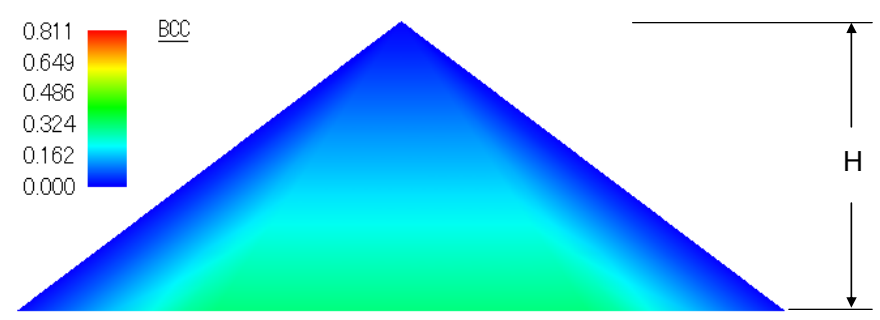

Fig.7a Distribution of normalized horizontal stress $\sigma_{x x} / \gamma \mathrm{H}$ under the BCC closure

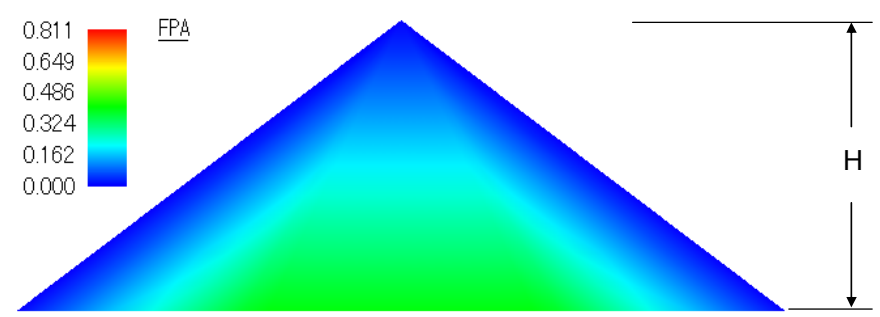

Fig.7b Distribution of normalized horizontal stress $\sigma_{x x} / \gamma \mathrm{H}$ under the FPA closure

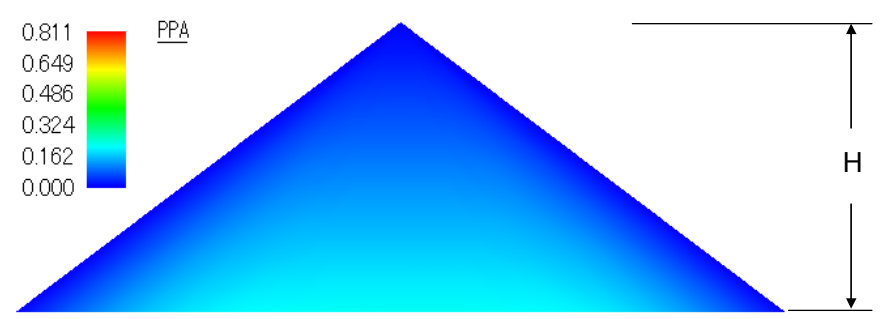

Fig.7c Distribution of normalized horizontal stress $\sigma_{x x} / \gamma \mathrm{H}$ under the PPA closure

According to Eq.(32), the color contour of normalized vertical stresses distribution for $\phi=37^{\circ}$ can be illustrated in following figures for the closures of BCC, FPA and PPA respectively.

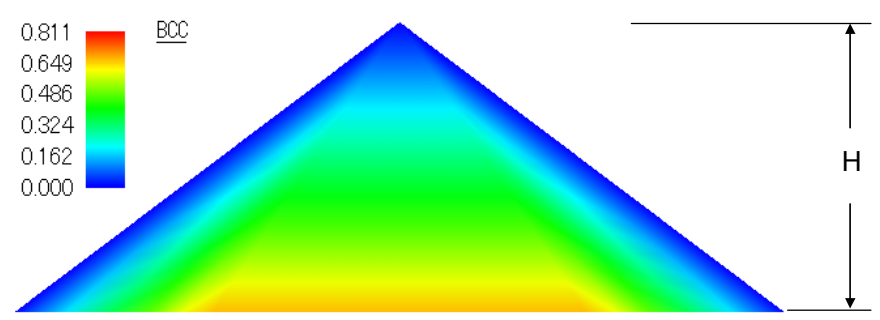

Fig.8a Distribution of normalized vertical stress $\sigma_{z z} / \gamma \mathrm{H}$ under the BCC closure

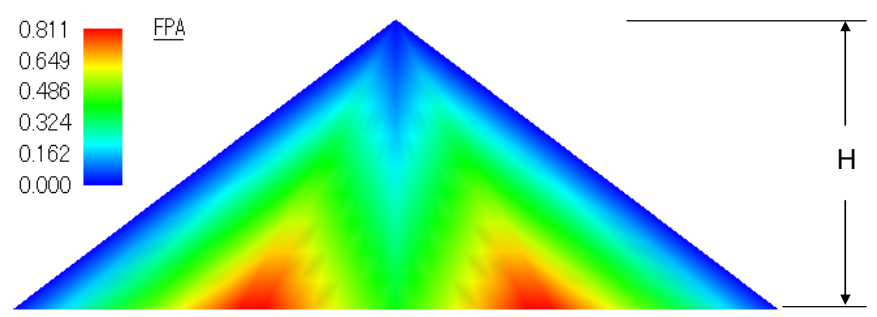

Fig.8b Distribution of normalized vertical stress $\sigma_{z z} / \gamma \mathrm{H}$ under the FPA closure 


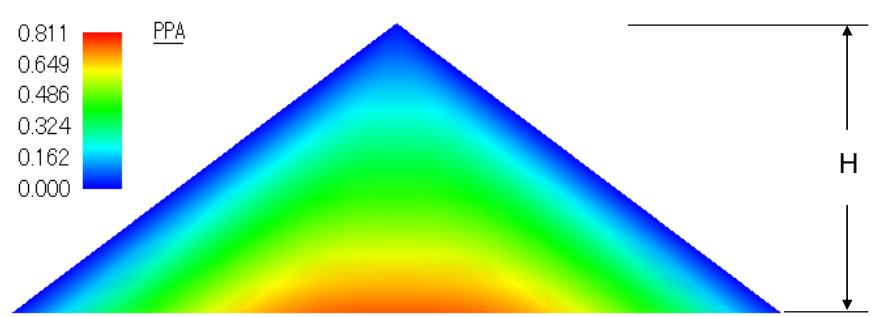

Fig.8c Distribution of normalized vertical stress $\sigma_{z z} / \gamma \mathrm{H}$ under the PPA closure

According to Eq.(32), the color contour of normalized shear stresses distribution for $\phi=37^{\circ}$ can be illustrated in following figures for the closures of BCC, FPA and PPA respectively.

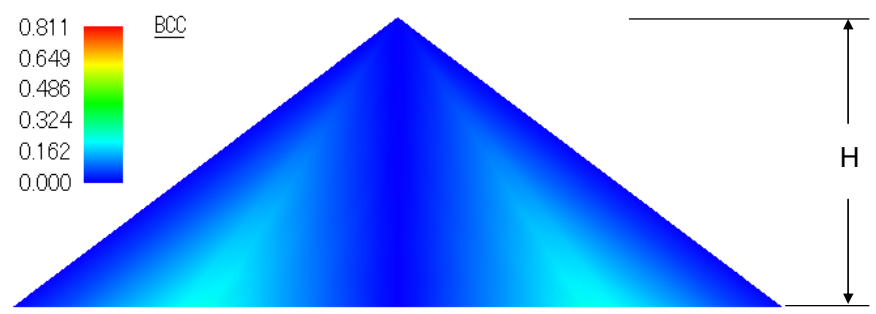

Fig.9a Distribution of normalized shear stress $\sigma_{x z} / \gamma \mathrm{H}$ under the BCC closure

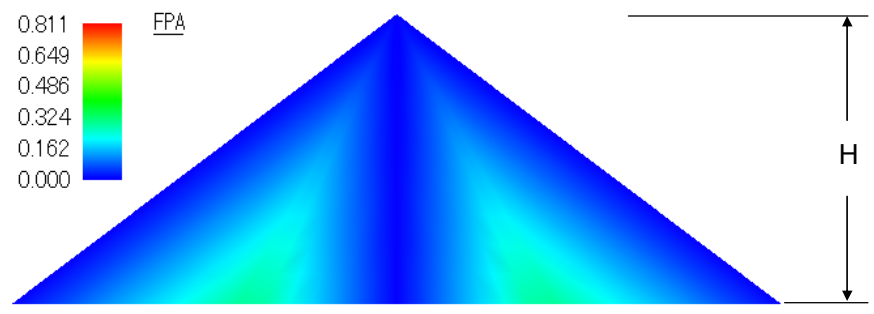

Fig.9b Distribution of normalized shear stress $\sigma_{x z} / \gamma \mathrm{H}$ under the FPA closure

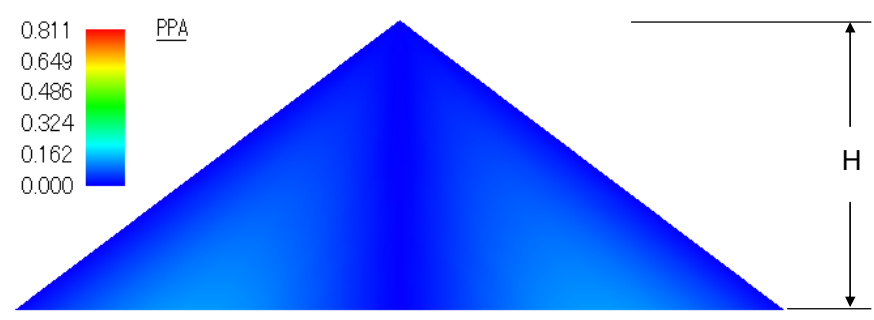

Fig.9c Distribution of normalized shear stress $\sigma_{x z} / \gamma \mathrm{H}$ under the PPA closure

\subsection{Arch shapes of self-weight transfer}

As with the linearity models, the solutions are separated into inner elastic and outer plastic regions which merge at a certain slope, therefore piecewise linear stress profiles are obtained. Stress solutions under the closures of oriented stress linearity provided by Wittmer et al. ${ }^{(5)}$ covers those of constant stress ratio and fixed principal axes whose directions of principal stress axes are illustrated in Figs.10a and $10 \mathrm{~b}$ using $\phi=37^{\circ}$ with unit height. Principal stress directions shown in Fig.10c are illustrated for the solution obtained under the closure of polarized principal axes. It can be observed in Fig.10c that self-weight is propagated along nonlinear load-bearing structures with a hump profile over the width of granular heaps. Moreover, plastic region is saturated only along the slope surface, therefore, smooth transition of arches transferring own weight can be noticed. The illustration of minor and major principal stress axes are shown in following figures, 


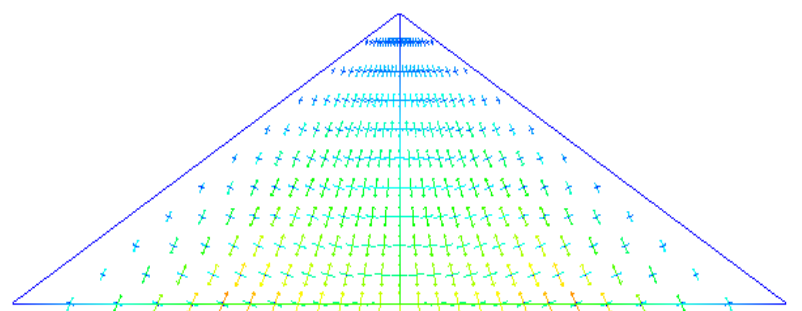

Fig.10a Directions of principal stress axes under the BCC closure

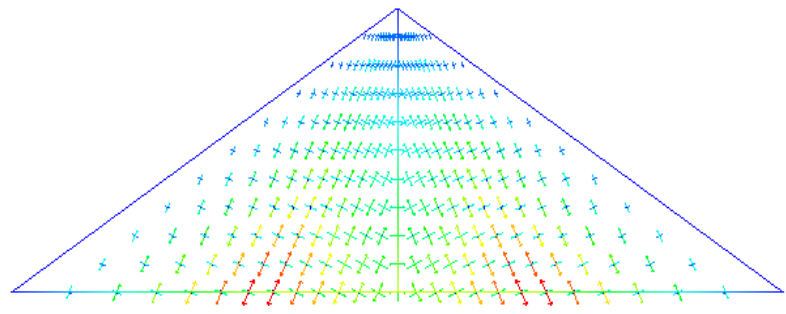

Fig.10b Directions of principal stress axes under the FPA closure

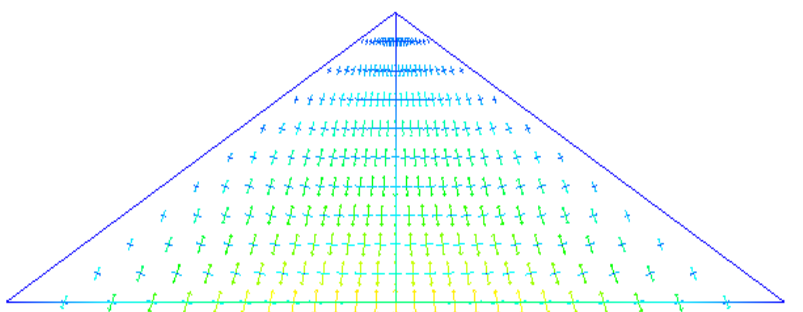

Fig.10c Directions of principal stress axes under the PPA closure

\subsection{Distribution of safety factor in heap body}

In continuum mechanics, granular materials are generally modeled as being elastic-plastic materials characterized by the failure condition. The classical Mohr-Coulomb failure criterion is widely accepted. This condition postulates that, each point every surface within the material, the ratio of deviatoric-to-mean stress satisfied the inequality where $q$ is a deviatoric stress and $p$ is a mean stress.

$$
\frac{q}{p}=\frac{\sqrt{\left(\sigma_{z z}-\sigma_{x x}\right)^{2}+4 \sigma_{x z}^{2}}}{\sigma_{z z}+\sigma_{x x}} \leq \sin \phi
$$

By substituting Eqs.(20)-(22) into Eq.(33), spatial distributions of safety factor $S F$ against sliding in loose heap of sand can be formulated to Eq.(34) as a ratio of resistance $p \sin \phi$ to deviatoric stress $q . S F$ is expressed in terms of scaling stresses which are related only to the relative aspect ratio $\bar{x} / z$ and aspect ratio $x / z$ for the BCC and FPA closures and with only the aspect ratio $x / z$ for the PPA closure.

$$
S F=\frac{\left(\sigma_{z z}+\sigma_{x x}\right) \sin \phi}{\sqrt{\left(\sigma_{z z}-\sigma_{x x}\right)^{2}+4{\sigma_{x z}}^{2}}}=\frac{\left(\chi_{z}+\chi_{x}\right) \sin \phi}{\sqrt{\left(\chi_{z}-\chi_{x}\right)^{2}+4 \chi_{x z}^{2}}} \geq 1
$$

$S F \geq 1$ represents the state of admissible stress which includes the failure state where $S F=1$. High value of $S F$ signifies high stability against sliding in sand heap since the corresponding admissible stress is far from the fully mobilized friction. By definition, $S F=1$ along the slope surface of sand heap in all closures because the stress condition saturates the Mohr-Coulomb failure criterion at the free-surface boundary.

With reference to the material used in heap construction, the friction angle of sand estimated from the constant-volume direct box-shear box test, $\phi=37^{\circ}$ is employed as the input parameter and the color contour of $S F$ distribution inside the heap reposed at this angle can be shown as follows. 


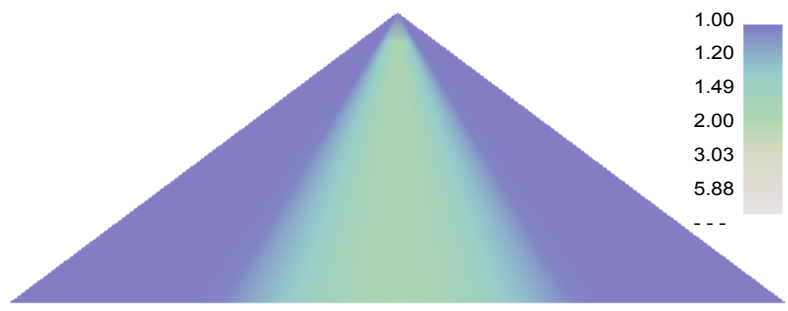

Fig. 11a Distributions of safety factor against sliding in loose heap of sand under the constant horizontal-to-vertical stress ratio (BCC) closure

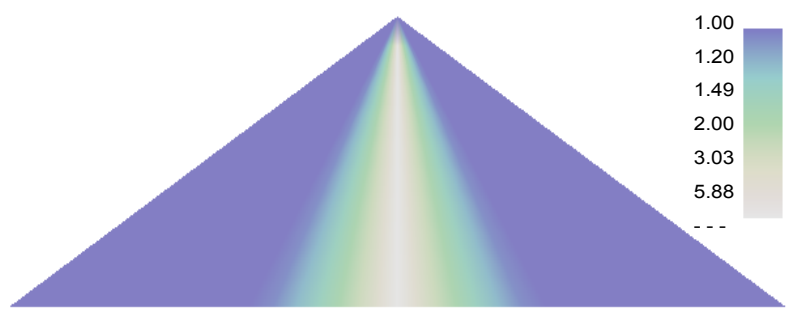

Fig. 11b Distributions of safety factor against sliding in loose heap of sand under the Fixed Principal Axes (FPA) closure

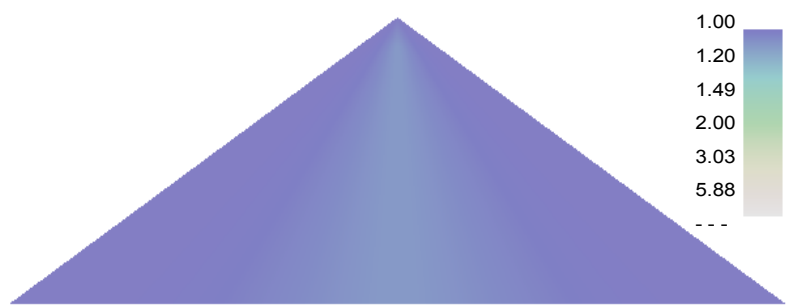

Fig. 11c Distributions of safety factor against sliding in loose heap of sand under the Polarized Principal Axes (PPA) closure

Figure 11a shows a deeper plastic zone in the BCC closure laid from the slope surface and separated with an elastic zone by a boundary from which the horizontal and vertical stress were saturated and formed two straight lines of elastic-plastic boundary passing the apex in both sides of sand heap. The safety factor against sliding of particles in that zone is equal unity which shows that particles in the zone are on the verge of flowing. The distribution of safety factor under the FPA closure shown by Fig.11b is in similar appearance with Fig.11a in which the elastic and plastic zones are clearly distinguished. However, the plastic zone in this model is wider and deeper. Figure 11c shows a plastic zone which lays just along the slope surface and confines the entire elastic zone. The safety factor at this surface is unity. As the safety factor against sliding gradually increases from unity from the surface to the center, the deeper sand lying below the heap surface remains stable and immobilized.

\section{Conclusions}

This study shows that stress field solutions under nature's law of friction can be derived through wave-like equations. Linear and nonlinear stress propagations due self-weight transfer in cohesionless granular media were investigated. Nests of principal stresses were illustrated and compared thru various stress conditions. It was found that the linear stress propagation follows straight arches with a certain elastic-plastic slope inside, while the nonlinear stress propagation follows the smooth curves of arches with elastic-plastic slope 
along the slope surface.

A deeper failure zone adjoining the slope surface within the body of heap characterized by the BCC and the FPA closures reveals that sand heap can be destabilized when the extra perturbation is applied. However, the PPA closure provides more reasonable basis that the body heap stays firm with its shape at the angle of repose. Steady sand heap as observed from the experiment is stable enough to maintain its shape and will not fail deeper inside its body. Careful experimental studies are required to verify this surprising region of failure which probably depends on methods of sand deposition.

\section{References}

(1) Hummel, F.H. and Finnan, E.J.: The distribution of pressure on surfaces supporting a mass of granular material. Minutes of the Proceedings of the Institution of Civil Engineers 212 (session 1920-1921 - PART 2 ), 369 - 392 (1921)

(2) Bouchaud, J.P., Cates, M.E. and Claudin, P.: Stress-distribution in granular media and nonlinear-wave equation. Journal De Physique I 5(6), 639-656 (1995)

(3) Bouchaud, J.P., Claudin, P., Cates, M.E. and Wittmer, J.P.: Models of stress propagation in granular media. Physics of dry granular media NATO ASI, Ser. E, Vol. 350, Cargèse, France, 97-121 (1997)

(4) Wittmer, J.P., Claudin, P., Cates, M.E. and Bouchaud, J.P.: An explanation for the central stress minimum in sand piles. Nature 382(6589), 336-338 (1996)

(5) Wittmer, J.P., Cates, M.E. and Claudin, P.: Stress propagation and arching in static sandpiles. Journal De Physique I 7(1), 39-80 (1997)

(6) Pipatpongsa, T, Heng, S., Modeling of self-weight transmission in prismatic granular heaps using continuum mechanics approach. In the 4th Asian Particle Technology Symposium, Delhi, CD-ROM: APT2009/154 (2009)

(7) Pipatpongsa, T., Iizuka, A. and Ohta, H., Analyses of pressure distribution using arching criteria in 2D sand heaps. In Proceedings of Theoretical and Applied Mechanics Japan, vol. 58, 41-48 (2010)

(8) Pipatpongsa, T, Heng, S., Iizuka, A and Ohta, H., Statics of loose triangular embankment under Nadai's sand hill analogy, Journal of the Mechanics and Physics of Solids, doi:10.1016/j.jmps.2010.07.013

(9) Sokolovskii, V.V.: Statics of Granular Materials, English translation edn. Pergamon Press, Oxford (1965)

(10) Nadai, A.: Theory of Flow and Fracture of Solids, McGraw-Hill, Part IV: Theory of Perfectly Loose and of Generally Plastic Substance, 435-506 (1963)

(11) Vanel, L., Howell, D., Clark, D., Behringer, R.P. and Clement, E.: Memories in sand: Experimental tests of construction history on stress distributions under sandpiles. Physical Review E 60(5), R5040-R5043 (1999) 